\title{
Beliefs In Advance Care Planning Among Chinese Americans: Similarities And Differences Between The Younger And Older Generations
}

Asian/Pacific Island Nursing Journal

Volume 3(2): 83-90

CAuthor(s) 2017

http://digitalscholarship.unlv.edu/apin/

\author{
Mei Ching Lee ${ }^{a}$, Ha Do Byon ${ }^{b}$, Katherine A. Hinderer ${ }^{c}$, and Carla Alexanderd
}

\begin{abstract}
The purpose of this research is to explore behavioral, normative, and control beliefs in the discussion of advance care planning (ACP) among older and younger Chinese Americans. Ethnic minority groups have been identified as less engaged in ACP and this represents an ethnic and cultural gap. Older Chinese American adults often have different beliefs and values compared to the younger generation who are more acculturated to American mainstream culture. These differences may hinder the discussion of ACP with Chinese older adults.

A qualitative design was used. The Theory of Planned Behavior guided the development of the interview guide. We recruited 60 Chinese Americans. Prior experience was identified as a theme that influenced attitudes about ACP. We found that older and younger Chinese participants had different beliefs in the norm and control related to ACP discussions, but not in the belief of attitudes about ACP discussions. Both younger and older Chinese American participants believed that ACP was important and necessary. Participants in both clusters expressed that they were ready and willing to engage in ACP discussions with their family members but hesitant to initiate these discussions. The reluctance in discussing ACP with Chinese older adults may be related to the expectations and obligations of Xiao (filial piety) in Chinese culture. This study describes the similarities and differences of beliefs in ACP between older and younger Chinese Americans. We identified barriers and facilitators in behavioral, normative, and control beliefs that can be used to promote ACP for Chinese Americans.
\end{abstract}

Keywords: advance care planning, Chinese culture, generation differences, filial piety, end-of-life care

Advance care planning (ACP) is a safeguard to acknowledge individuals' autonomy. It is a process of communication and discussion of future health care planning that includes setting goals, making health care decisions, and appointing surrogate decision makers (U. S. Department of Health and Human Services, 2008; Zhang et al., 2015). Planning is not only for people at an older age or with a terminal illness who are admitted to the hospital. Healthy people in the community should also make plans for future health care, including expressing their values and their end-of-life (EOL) wishes (National Institute on Aging, 2015). Research shows ACP is beneficial to both patients and their family members. It improves patients' quality of life by assisting them in recognizing their goals and preparing for EOL. It reduces family members' psychological burden when they need to make decisions for patients by providing them with reassurance and confirmation of their loved ones' wishes (Centers for Disease Control and Prevention, 2013). However, ethnic minority groups are less engaged, showing ethnic and cultural gaps in engagement in ACP (Pecanac \& Repenshek, 2012). The purpose of this study was to explore the beliefs, facilitators, and barriers of ACP discussion among different generations of Chinese Americans.

\footnotetext{
${ }^{a}$ University of Maryland at Baltimore, USA

${ }^{b}$ University of Virginia, USA

${ }^{c}$ Salisbury University, USA

${ }^{d}$ University of Maryland, USA
}

Corresponding Author:

Mei Ching Lee, PhD, MS, RN

School of Nursing

University of Maryland

Email: mclee@umaryland.edu 


\section{Intergeneration Differences and $A C P$ in Chinese Americans}

Chinese Americans represent the largest Asian group in the United States. In a 2013 American Community Survey, there were 4.3 million Chinese Americans, and this number is rapidly growing (Wong, 2013). Among all Chinese Americans, threequarters $(76 \%)$ of adults are foreign-born (United States Census Bureau, 2010), with values and beliefs strongly shaped and influenced by the traditional Chinese culture. These cultural beliefs may conflict with the over-arching American culture of ACP (Chew, 2011). In traditional Chinese culture, the value of an individuals' autonomy and the discussion of ACP is regarded differently compared to the U.S. culture (Oyserman \& Lee, 2008). Traditional Chinese believe that family and society are the foundation of the individuals' existence and value the collectivism of family and society in making health care decisions more than individualism of decision-making. Moreover, they believe open discussions to prepare for the dying process will hasten death, making the discussion of ACP a taboo (Wong, 2013). On the other hand, their children, who are acculturated to the America mainstream culture, tend to accept the values of autonomy, individuality, and self-regulation. Thus, they have less cultural burden in the discussion of death and dying (Koepke \& Denissen, 2012). These differences in values and beliefs may create dilemmas in the discussion of ACP between Chinese parents and children. Furthermore, Xiao, or filial piety, confronts Chinese Americans in both younger and older generations. Xiao is a highly valued family virtue in Chinese culture. It dictates the expectations and obligations of children. Children are obligated to respect, care, greet, obey, and assure their parents' physical and emotional well-being (Yu et al., 2016; Dong, Zhang, \& Simon, 2014). Discussing taboo topics such as ACP is viewed as upsetting, uncaring, and disrespectful to others. Therefore, the process of discussing and planning for EOL, such as ACP, is not compatible with the cultural beliefs of Xiao.

A systematic review by Lin, Bryant, Boldero, and Dow (2015) used search terms of Chinese, older adults, family relationships, filial piety, and conflicts to explore the relationship between Chinese parents and children and concluded that intergenerational differences exist in traditional values and beliefs. These conflicts and confusion between generations may hinder honest communication of ACP among Chinese family members. A better understanding of ACP beliefs in Chinese Americans of different generations would provide valuable information for the development of a culturally sensitive approach to promote ACP in the Chinese American community.

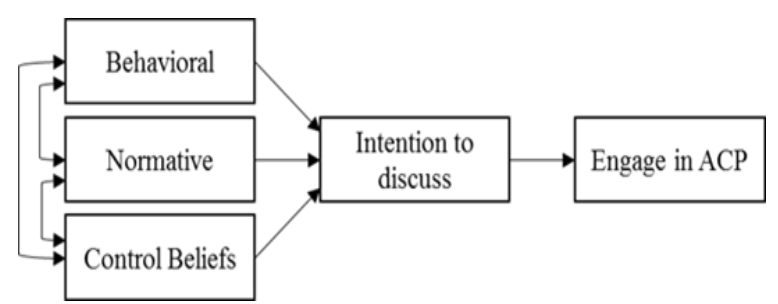

Figure 1. Theory of Planned Behavior in advance care planning (ACP) modified from the Theory of Planned Behavior (Ajzen, 2017).

\section{Theory of Planned Behavior (TPB)}

The TPB is a predictive theory suggesting that behaviors are the outcomes of a set of selfperceived beliefs. According to the TPB framework, ACP is a health behavior that is motivated when people believe that ACP is beneficial (Behavioral Beliefs), desirable (Normative Beliefs), and achievable (Control Beliefs; Ajzen, 2017; Figure 1). We intended to explore these beliefs among both Chinese American parents and children to further understand the facilitators and barriers of engaging in ACP.

\section{Method}

We used a qualitative descriptive design for the focus group discussions. The group interview method was selected for this study, as it was less expensive compared with individual interviews, but more inclusive of information than closed-ended questions in a survey. Also, the observation of group interaction and the non-verbal communication provided added insights on the study topic (Morgan, 1997). A semi-structured discussion guide was developed using the framework of TPB to explore the three core beliefs of behavioral, normative, and control that mediate the discussion of ACP in Chinese American older adults (Ajzen, 2017).

\section{Sample}

We recruited a convenience sample of 60 community-dwelling Chinese Americans from a Chinese Community Center located in a metropolitan area in the mid-Atlantic region of the United States. The inclusion criteria were adults who were above 18 years old, self-identified as Chinese American, and spoke either Mandarin, Cantonese, or English. We divided the sixty participants into two groups by age. We grouped participants aged 65 years or above $(n=$ 30 ) in the older group (OG). All participants in this group had at least one chronic disease that required regular physician visits and prescribed medications. We placed participants who were below 65 years old 
Lee et al.: Beliefs In AdvanceCare Planning A mong Chinese Americans

Table 1. Similarities and Differences of Beliefs in ACP between Older and Younger Chinese Americans $(N=60)$.

\begin{tabular}{|c|c|c|}
\hline \multirow[b]{2}{*}{ Core questions } & \multicolumn{2}{|c|}{ Themes } \\
\hline & OG & YG \\
\hline $\begin{array}{l}\text { Behavioral Beliefs: } \\
\text { Is ACP important or not } \\
\text { Important and why? }\end{array}$ & - $\quad$ ACP is good and needed & - $\mathrm{ACP}$ is important \\
\hline $\begin{array}{l}\text { Normative Beliefs: } \\
\text { What do you think the experience } \\
\text { would look like if you discuss } \\
\text { ACP with your family/friends? } \\
\text { Do most of the people who are } \\
\text { important to you think ACP } \\
\text { should be done/should not be } \\
\text { done? }\end{array}$ & $\begin{array}{l}\text { - } \\
\text { - Children discussion is a taboo } \\
\text { cuss ACP } \\
\text { - Discussing ACP with children } \\
\text { will upset them }\end{array}$ & $\begin{array}{l}\text { - } \text { ACP discussion is a taboo } \\
\text { - Not supported by the Chinese socie- } \\
\text { ty } \\
\text { - Easier to have ACP conversation } \\
\text { with younger generation } \\
\text { - Discussing ACP with parents will } \\
\text { be criticized, labeled as no filial and } \\
\text { disrespectful }\end{array}$ \\
\hline $\begin{array}{l}\text { Control Beliefs: } \\
\text { What makes it easy or enables } \\
\text { you to have the discussion of ACP } \\
\text { with your family/friends? } \\
\text { What makes it difficult or pre- } \\
\text { vents you from having the discus- } \\
\text { sion of ACP with your fami- } \\
\text { ly/friends? } \\
\text { Do you think you could bring up } \\
\text { the topic of ACP with your fami- } \\
\text { ly/friends? Why and how? }\end{array}$ & $\begin{array}{l}\text { - } \\
\text { - } \quad \text { Lack of capable to discuss ACP } \\
\text { ACP } \\
\text { - Lack of English proficiency } \\
\text { Lack of AD tool that is sensi- } \\
\text { tive to Chinese culture. }\end{array}$ & $\begin{array}{l}\text { - } \quad \text { Not capable to discuss ACP } \\
\text { - Afraid to be a surrogate and make } \\
\text { wrong decisions } \\
\text { - Being a surrogate decision maker is } \\
\text { a huge responsibility }\end{array}$ \\
\hline
\end{tabular}

Note . $\mathrm{ACP}=$ advance care planning; $\mathrm{AD}=$ advance directive; $\mathrm{OG}=$ older group; $\mathrm{YG}=$ younger group

$(n=30)$ in the younger group (YG). All participants in this group had at least one elderly parent who required their attention or support. We conducted ten focus group discussions: five focus group discussions for each OG and YG. Each group had 2-7 participants. We purposefully divided the sample into two groups to examine the similarities and differences of beliefs and cultural implications in ACP among different generations.

\section{Ethical Considerations}

The Institutional Review Board of the University of Maryland Baltimore (HP-00058059) approved this study. We explained the purpose of the study, risks, and potential benefits to all potential participants. Participants were informed of the voluntary nature of the study and were aware they could withdraw from the study at any time. The consent form was written in Chinese and English language. We obtained the informed consent before data collection.

\section{Data collection}

We used a semi-structured interview guide for each of the 10 focus group sessions. One of the authors conducted all interviews. We conducted all focus group discussions in Mandarin except one group (conducted in Cantonese) due to participants' language preferences. Each focus group lasted about 2 hours. All sessions were audiotaped. Once data collection was completed, we sent the audiotapes to a professional translation and transcription company that translated Chinese into English and transcribed all of the recorded interviews.

\section{Data Analysis}

The Framework Method for analysis of qualitative data with both deductive and inductive approaches was used (Gale, Heath, Cameron, Rashid, $\&$ Redwood, 2013). The TPB provided an analytical and interpretive framework of its three constructs (behavioral, normative and control beliefs) within which identified themes could be structured (deductive). However, open coding was also employed to allow for independent groups of themes to develop beyond the three constructs to ensure unexpected, important aspects of the data were not missed (inductive). Data analysis started when data collection began. Two of the authors independently read the textual data from the first few transcripts and developed an initial working analytical framework composed of 
Table 2. Enablers and Barriers to the ACP among Chinese Americans $(N=60)$.

\begin{tabular}{ll}
\hline Enablers to ACP discussion & Barriers to ACP discussion \\
\hline Prior experience related to death and dying (both groups) & Knowledge of ACP (OG) \\
Community outreach programs (both groups) & Language barriers (OG) \\
Indirect approach (OG) & Burdens of making decisions (YG) \\
& Lack of appropriate opportunity for ACP discussion (both \\
& groups) \\
& Cultural sensitive AD (OG) \\
\hline
\end{tabular}

Note $. \mathrm{ACP}=$ advance care planning; $\mathrm{AD}=$ advance directive; $\mathrm{OG}=$ older group; $\mathrm{YG}=$ younger group

agreed-upon codes and categories. The working analytical framework was iteratively reviewed and refined by three authors until the last transcript was coded. A thematic approach guided the line-by-line analysis of the transcripts (van Manen, 1990). We grouped codes into categories around similar and interrelated concepts, and identified themes by integrating the categories. We also examined the commonalities and differences between the two participant groups. NVivo software was used to code and analyze the data.

\section{Rigor}

We used Lincoln and Guba's (1985) criteria to establish trustworthiness of the qualitative study. First, we conducted all interviews in the participant's language of choice, Mandarin, Cantonese, or English. Participants were grouped into focus groups according to language preference so they could express themselves adequately. Second, we provided the study design and methods for reference and replication. Third, the discussion and description of the findings facilitated the use of the results in other populations. A team approach was used in data analysis to promote non-bias interpretation of data. A discussion guide was used for all interviews to maintain consistency. We audiotaped all focus group discussions. The tapes were sent to a professional translation and transcription company to translate all interviews into English. The audio recordings were then transcribed and provided to the authors who then reviewed them for subsequent analysis and coding.

\section{Results}

The sample had a mean age of $53(S D=$ 18.33), range from 29 to 79 years old. Three-quarters of the sample $(75 \%)$ were females. Table 1 summarizes the identified themes.

\section{Behavioral Beliefs}

Behavioral beliefs described participants' beliefs regarding the purpose of ACP discussions.
Individuals that have positive attitudes about ACP believed that ACP was important, necessary, and produced good outcomes for patients and their families. Most of the participants in the OG and YG had positive attitudes towards ACP. Both the elderly parents and adult children were accepting of ACP, believed that ACP was essential for care and that it was needed for people regardless of their health status:

- "Important. Very important. Of course it is important." (YG)

- "It is not only important to the elderly people, it is important to us all here as well." (YG)

- "I think it is good. I think that idea is very good." (OG)

- "It's not just something that we can discuss, but also something we should do." (OG)

The most common desired outcome of ACP in both the OG and YG was that it lessened the burden of others in making EOL decisions for them:

- "I have spoken to my son about this because I don't want to burden him."(OG)

- "They might ask why you decided not to save your parents when it comes to that moment. If we go along with the Chinese tradition, then it would be too much trouble for the children. It will not be a trouble for your children if you make it clear." (OG)

- "It helps family members to mentally prepare what to do. If there is no ACP, we wouldn't know anything...." (YG)

- "It is not only important for oneself, it is also important for family members... At least we could tell what she [my mom] is thinking about." (YG)

Participants in both groups mentioned that prior experiences related to death and dying changed their attitudes toward ACP:

- "Because she [my mom] had the experience... after my Dad died... she always talks about how she definitely doesn't want to be in a vegetable state, nor have the tubes. (YG)

- "Having that experience, they all went to the lawyers' and made the will. They didn't 
want anything like that happen to them in the future." (YG)

- "This is because the death of my son. I saw him suffering when he was at the hospital. It was suffering for him to go through the rescuing measures and chemotherapy. So we have told our grandson, my wife, and also our daughter when she was here, we have told someone from our church." (OG)

- "We saw friends suffering before. We saw them with a lot of tubes. We felt really upset. The tubes are not necessary. Our children understand this [our plan] as well." (OG)

\section{Normative Beliefs}

Normative beliefs described participants' beliefs regarding social expectations of discussing ACP in the Chinese culture. Participants in both groups, YG and OG, believed that the discussion of death and dying and the planning for EOL care is a taboo in the Chinese society. The YG perceived that the discussion of ACP with the OG would be unpleasant and difficult, and therefore, would upset the senior members of the family and society:

- “They [older generation] won't like talking about this issue [ACP]. The young people are scared of mentioning it ... no one wants to be the 'bad guy.' If you said it first, the other people will criticize you. Other relatives will criticize you."

- "They will think they have brought us up, and it is our responsibility to look after them. How dare you talk about something so sensitive? Do you want us to die soon? If I mention this, my Dad would be angry straight away."

- "She [parent] would say you curse her to death."

- "Yes, difficult. I feel it is disrespectful to talk to the elderly about this [ACP]."

However, participants in the $Y G$ found it easier to have conversations about ACP among their generation or with their children:

- "We don't have any problem [discussing ACP] with our children."

- "It is easier to talk to the younger generation [about ACP]."

- "Our generation, yes [to discuss ACP]. The generation above us, rarely."

Participants in the OG acknowledged that the cultural belief of discussing death and dying is a taboo. They also perceived that the discussion of ACP would be unwelcome and upsetting to their children:
- "The Americans would talk about it in advance. But it is a taboo topic for Chinese."

- "It [ACP] is not allowed to be talked about or mentioned."

- "I can't talk about it [ACP] with my children."

- "The children don't really like talking about this [ACP] with you."

\section{Control Beliefs}

Control beliefs described participants' beliefs regarding the ability to have an ACP discussion. Participants in the OG expressed lack of knowledge about ACP. Some of them had never heard of ACP, and confused this with making a will, euthanasia, or making a funeral arrangement. When the concept of ACP was clarified, participants in this group believed that it was difficult to discuss ACP because they lacked personal support and necessary materials such as specific ACP information, Chinese language support, appropriate translated forms, and counseling services:

- "Is it [ACP] new? I didn't know it before. It is quite new to me."

- "I have never heard of it."

- "I think discussing [ACP] in Chinese is definitely more comfortable. No matter how good your English is...If you speak English better, then discuss in English. If you prefer Cantonese, then discuss in Cantonese."

- "I just let it pass if I hear [English] words that I don't understand. [laughter]"

- "It's easier to communicate [ACP] with Chinese speakers. This is the most important thing. I don't want the messengers... it is not the same thing."

- "What shall I do after I write it [ACP]? I don't need to take it to the court, right?"

- "It is better if you can design a format for this kind of will. Probably it's better to have a form, so we can fill out the form, instead of writing things on our own...."

Participants in the YG faced a different kind of challenge in discussing ACP with their parents. The challenges were mostly related to being the surrogate, the interpreter of decision-making. They felt conflicted because they did not know their parents' wishes:

- "We are scared of making decisions."

- "There will be a lot of pressure."

- "No one wants the blame. There will be different opinions [among the children]."

Participants in both groups expressed that the biggest barrier to holding an ACP discussion is lack of an appropriate opportunity: 
- "You have to find the appropriate moment when they are in a good mood." (YG)

- "We need an appropriate [safe] opportunity." (YG)

- "I just never found the right moment to initiate the conversation." (YG)

- "They [children] are too busy; they have very little time to spend with me."(OG)

\section{Resources and Support to Encourage ACP discussion}

In regards to the resources and support that will help participants with ACP discussions, most of the participants suggested using community outreach programs; they particularly liked the outreach formats of a seminar or group discussion. They suggested using a case scenario, and video sharing that is not too personal-oriented to introduce the discussion. The other suggestion was to create opportunities for parents and children to get together to discuss this issue. Participants believed that making an advance directive that is written in Chinese and is sensitive to their specific cultural needs would increase their acceptance of ACP and the use of advance directive (Table 2).

- "We can organize a lecture, so the people will talk about and discuss it together. Like us now. They can discuss what they think if they go through a similar situation. It is better to get it done gradually." (YG)

- "Get some people to share their experience, and tell the elderly people that they have gone through this kind of situation. It would be more convincing in this way. It makes it interesting to the elderly people. And then let them know that we are available to assist them too." (YG)

- "If you don't talk about the content and only give out some paperwork, then it will be binned after they go home. Also, books or flyers are equally useless." (OG)

- "We can discuss it in a small group because it is a serious matter." $(\mathrm{OG})$

- "....use case study that will not be too personal.” (OG)

\section{Discussion}

In this study, almost all of the participants believed ACP was important and necessary. The receptivity to ACP discussions in the OG was somewhat surprising. A study suggested that older, less acculturated minorities do not see the benefit in ACP (Gao, Sun, Ko, Kwak, \& Shen, 2015; Kwak \& Haley,
2005; Matsumura et al., 2002). The finding that older participants had a positive attitude about ACP in this study was encouraging: despite the traditional cultural beliefs about death and dying, introducing and promoting the ACP in Chinese Americans could be feasible and practical.

This study revealed an interesting intergenerational phenomenon likely caused by social expectations and cultural beliefs. To be prepared, participants in the YG wanted to know their parents' EOL wishes. However, they were afraid to initiate the discussion, as they believed that ACP was a taboo in the Chinese American society and their parents would be upset if they discussed it. They worried that their parents would view the initiation of ACP as acting against filial piety and hastening death. On the other hand, participants in the OG stated that they wanted to make their wishes known to their children so that their children could arrange their EOL care without any doubt or guilt. However, they did not initiate the discussion, as they believed their children would be uncomfortable discussing ACP with them. The elders worried that their children would feel blamed for not having filial piety if they initiated an ACP discussion. The findings of hesitation in ACP discussion among Chinese Americans were consistent with the existing literature describing the topic of death and dying as a taboo in the Chinese society. Chinese believe that discussing these topics will bring bad luck to individuals, and conversation related to death is considered disrespectful. In addition, any planning surrounding death is believed to hasten death and is therefore inappropriate to discuss (Lee, Hinderer, \& Kehl, 2014; Wong, 2013; Zou, O'Conner, Peters, \& Jiejun, 2013). However, younger Chinese Americans in this study found it easier to discuss the ACP with people of the same generation. More research is needed to understand the role that intergenerational differences and Xiao play in the ACP discussions in Chinese Americans.

Older adults usually require more resources and support to engage in ACP discussions (Simon, Porterfield, Bouchal, \& Heyland, 2015). This finding is consistent with the findings in our study. Older Chinese participants in this study expressed needing more assistance with ACP such as access to information, interpretation, and translation of advance directives, compared to younger participants. Resources and support to promote ACP discussion should be more focused on the older population.

One of the identified barriers to ACP discussions in the OG and YG was having a safe opportunity to initiate the discussion. Participants in the OG and YG stressed that ACP was good, but they were reluctant to initiate the discussion. Although many studies were conducted to provide recommendations 
and step-by-step guides for ACP discussion (Fried, 2009; National Hospice and Palliative Care Organization, 2016; The Conversation Project, 2017; Sudore et al., 2008; Westley \& Briggs, 2004), limited work has been done specifically for Chinese Americans. Research is needed to create culturally appropriate opportunities for holding ACP discussions for Chinese Americans.

Having prior experience with death and dying was identified as an enabler to motivate the discussion of ACP. Prior experience is not one of the constructs in the TPB. However, the impact of prior experience in predicting human behaviors is discussed widely in the literature (Kim, \& Choi, 2016; Sommer, 2011). Further studies are needed to determine whether prior experience is a predictive factor in facilitating ACP discussions among Chinese Americans. Other facilitators identified in this study included the format and approach to promoting ACP discussion. Participants expressed that using small group or case study formats and an indirect approach will make ACP discussions easier. Future research may incorporate these findings to develop effective interventions to promote ACP discussion in Chinese Americans.

\section{Conclusions}

Both younger and older Chinese Americans in this study believed that ACP was important and necessary. They also believed that ACP produced good outcomes for patients and their family members. However, the majority of the participants found it difficult to initiate ACP conversations. Participants in the YG found the conversation of ACP much more acceptable among their generation compared to the older generation. The differences may be because the younger generation had fewer burdens related to cultural beliefs around death and dying, better English communication skills, and more knowledge of ACP. In this study, finding the right time and opportunity was one of the biggest barriers to initiating ACP discussions. Also, the expectations and obligations of Xiao may have influenced reluctance in discussing ACP with older Chinese adults. Choosing between fulfilling Xiao, not upsetting elders and parents, and discussing ACP in advance to understand parents' EOL wishes, was an extremely challenging dilemma for young Chinese Americans in this study. This study described the similarities and differences of beliefs about holding ACP discussions between older and younger Chinese Americans. We identified barriers and facilitators in beliefs of behavioral, normative, and control. In addition, we described the resources and support needed for each group, which can be used to promote ACP for Chinese Americans.

\section{Limitations and Strengths}

Given the qualitative design and nonrepresentative sample, these findings cannot be generalized to populations that are different from this sample. Acculturation may affect participants' beliefs in ACP regardless of their age and may introduce bias in the discussion. In this study, none of the participants in the OG were fluent in English, and very few of them knew of ACP. These findings reflect that older participants in this study were probably not highly acculturated. Future studies should include acculturation and other related variables that are related to ACP discussion in the design. Despite these limitations, the present study contributed to the literature by exploring the similarities and differences in beliefs about holding ACP discussions between older and younger Chinese Americans. We also identified barriers and facilitators in promoting ACP discussion for future research studies.

\section{Declaration of Conflicting Interests}

The authors do not have any financial interest or benefit from direct applications of their research.

\section{Funding}

This research was funded by the University of Maryland Baltimore, School of Nursing.

\section{References}

Ajzen, I. (2017). Theory of planned behavior. Retrieved from http://people.umass.edu/aizen/tpb.html

Centers for Disease Control and Prevention. (2013). Advance care planning: Ensuring your wishes are known and honored if you are unable to speak for yourself. Retrieved from http://www.cdc.gov/aging/pdf/advanced-careplanning-critical-issue-brief.pdf

Chew, P. K. (2011). A case of conflict of cultures: End-oflife decision making among Asian Americans. Cardozo Journal of Conflict Resolution, 13, 379$392 . \quad$ Retrieved from http://heinonline.org/HOL/LandingPage?handle= hein.journals/cardcore13\&div=18\&id=\&page

Dong, X., Zhang, M., \& Simon, M. A. (2014). The expectation and perceived receipt of filial piety among Chinese older adults in the Greater Chicago area. Journal of Aging and Health, 26, 1225-1247. https://doi.org/10.1177/0898264314541697

Fried, T. R., Bullock, K., Iannone, L., \& O’Leary, J. R. (2009). Understanding advance care planning as a process of health behavior change. Journal of the American Geriatrics Society, 57, 15471555. https://doi.org/10.1111/j.1532$\underline{5415.2009 .02396 . x}$ 
Gale, N., Heath, G., Cameron, E., Rashid, S., \& Redwood, S. (2013). Using the framework method for the analysis of qualitative data in multi-disciplinary health research. BMC Medical Research Methodology, 13(1). https://doi.org/10.1186/1471-228813-117

Gao, X., Sun, F., Ko, E., Kwak, J., \& Shen, H. -W. (2015). Knowledge of advance directive and perceptions of end-of-life care in Chinese-American elders: The role of acculturation. Palliative and Supportive Care, 13, 1677-1684 https://doi.org/10.1017/s147895151500067x

Kim, K. M., \& Choi, J. S. (2016). Mothers' intentions to vaccinate their teenaged children against human papillomavirus, as predicted by sex in South Korea: An application of the theory of planned behavior. Japan Journal of Nursing Science. https://doi.org/10.1111/ijns.12155

Koepke, S., \& Denissen, J. J. A. (2012). Dynamics of identity development and separation-individuation in parent-child relationships during adolescence and emerging adulthood-A conceptual integration. Developmental Review, 32, 67-88. https://doi.org/10.1016/j.dr.2012.01.001

Kwak, J., \& Haley, W. E. (2005). Current research findings on end-of-life decision making among racially or ethnically diverse groups. The Gerontologist, 45, 634-641. https://doi.org/10.1093/geront/45.5.634

Lee, M. C., Hinderer, K. A., Kehl, K. A. (2014). A systematic review of advance care planning and advance directives in Chinese people from Eastern and Western cultures. Journal of Hospice \& Palliative Nursing, 16, 75-85. https://doi.org/10.1097/njh.0000000000000024

Lin, X., Bryant, C., Boldero, J., \& Dow, B. (2015). Older Chinese Immigrants' relationships with their children: A literature review from a solidarityconflict perspective. The Gerontologist, 55, 9901005. https://doi.org/10.1093/geront/gnu004

Lincoln, Y. S., \& Guba, E. G. (1985). Naturalistic inquiry. Newbury Park, CA: Sage Publications.

Matsumura, S., Bito, S., Liu, H., Fukuhara, S., KagawaSinger, M., \& Wenger, N. (2002). Acculturation of attitudes toward end-of-life care: A crosscultural survey of Japanese Americans and Japanese. Journal of General Internal Medicine, 17, 531-539. $\quad$ https://doi.org/10.1046/j.15251497.2002.10734.x

Morgan, D. (1997). Focus group as qualitative research (2nd ed.). Thousand Oaks, CA: Sage Publications. http://dx.doi.org/10.4135/9781412984287

National Hospice and Palliative Care Organization. (2016). Communicating end-of-life wishes (Report No. 810001). Retrieved from http://www.caringinfo.org/files/public/brochures/ Communicating_EOL_Wishes.pdf

National Institute on Aging. (2015). Advance care planning. $\quad$ Retrieved from https://www.nia.nih.gov/health/publication/advan ce-care-planning

Oyserman, D., \& Lee, S. W. S. (2008). Does culture influence what and how we think? Effects of priming individualism and collectivism. Psychological Bulletin, 134, 311-342. https://doi.org/10.1037/0033-2909.134.2.311
Pecanac, K., \& Repenshek, M. (2012). Improving advance care planning in a racially and ethnically diverse community [abstract]. BMJ Supportive \& Palliative Care, $\quad 2, \quad 180$. https://doi.org/10.1136/bmjspcare-2012$\underline{000250.37}$

Simon, J., Porterfield, P., Bouchal, S. R., \& Heyland, D. (2015). 'Not yet' and 'Just ask': Barriers and facilitators to advance care planning-a qualitative descriptive study of the perspectives of seriously ill, older patients and their families. BMJ Supportive \& Palliative Care, 5, 54-62. https://doi.org/10.1136/bmjspcare-2013-000487

Sommer, L. (2011). The Theory of Planned Behavior and the impact of past behavior. International Business \& Economics Research Journal, 10, 91-110.

Sudore, R. L., Schickedanz, A. D., Landefeld, C. S., Williams, B. A., Lindquist, K., Pantilat, S. Z., \& Schillinger, D. (2008). Engagement in multiple steps of the advance care planning process: A descriptive study of diverse older adults. Journal of the American Geriatrics Society, 56, 1006-1013. https://doi.org/10.1111/j.15325415.2008.01701.x

The Conversation Project. (2017). Starter kits. Retrieved from http://theconversationproject.org/starterkits/

U. S. Department of Health and Human Services. (2008). Advance directives and advance care planning: Report to Congress. Retrieved from http://aspe.hhs.gov/daltcp/reports/2008/ADCong Rpt.pdf

United States Census Bureau. (2010). The foreign-born population in the United States: 2010. Retrieved from http://www.census.gov/prod/2012pubs/acs19.pdf

van Manen, M. (1990). Researching lived experience: Human science for an action sensitive pedagogy. New York: The State University of New York.

Westley, C., \& Briggs, L. A. (2004). Using the stages of change model to improve communication about advance care planning. Nursing Forum, 39(3), 5$12 . \quad$ https://doi.org/10.1111/j.17446198.2004.tb00003.x

Wong, D. (2013). Chinese ethics. In E. N. Zalta (Ed.), The Stanford encyclopedia of philosophy. Retrieved from http://plato.stanford.edu/archives/spr2013/entries/ ethics-chinese

Yu, H., Wu, L., Chen, S., Wu, Q., Yang, Y., \& Edwards, H. (2016). Caregiving burden and gain among adultchild caregivers caring for parents with dementia in China: The partial medicating role of reciprocal filial piety. International Psychogeriatric, 28, 1845-1855. https://doi.org/10.1017/s1041610216000685

Zhang, N., Ning, X., Zhu, M., Liu, X., Li, J., \& Liu, Q. (2015). Attitude towards advance care planning and healthcare autonomy among communitydwelling older adults in Beijing, China. BioMed Research International, 2015, 1-10. https://doi.org/10.1155/2015/453932

Zou, M., O’Conner, M., Peters, L., \& Jiejun, W. (2013). Palliative care in mainland China. Asia Pacific Journal of Health Management, 8, 9-13. 